\title{
An Energiser with Herbal Extracts, Creatine and Collagen to Prevent Post-Exercise Fatigue
}

\author{
Kwok-pui Fung ${ }^{1,2}$, Clara Bik-san Lau ${ }^{1,2}$, Ellie Suet-yee Pang ${ }^{1}$, William King-fai Cheng ${ }^{1}$, \\ Chun-kwok Wong ${ }^{1,2,3}$, Ping-chung Leung ${ }^{1,2, *}$ \\ ${ }^{1}$ Institute of Chinese Medicine, the Chinese University of Hong Kong, Hong Kong \\ ${ }^{2}$ State Key Laboratory of Phytochemistry and Plant Resources in West China, the Chinese University of Hong Kong, Hong Kong \\ ${ }^{3}$ Department of Chemical Pathology, the Chinese University of Hong Kong, Hong Kong
}

\section{Email address:}

kpfung@cuhk.edu.hk (K. P. Fung), claralau@cuhk.edu.hk (C. B. S. Lau), elliepang@cuhk.edu.hk (E. S. Y. Pang), kfcheng@cuhk.edu.hk (W. K. F. Cheng), ck-wong@cuhk.edu.hk (C. K. Wong), pingcleung@cuhk.edu.hk(P. C. Leung)

\section{To cite this article:}

Kwok-pui Fung, Clara Bik-san Lau, Ellie Suet-yee Pang, William King-fai Cheng, Chun-kwok Wong, Ping-chung Leung. An Energiser with Herbal Extracts, Creatine and Collagen to Prevent Post-Exercise Fatigue. International Journal of Nutrition and Food Sciences. Vol. 4, No. 6, 2015, pp. 639-643. doi: 10.11648/j.ijnfs.20150406.16

\begin{abstract}
Physical exercises are important for maintaining physical strength and general health state, particularly for the senior citizens. However, after strenuous exercises, the feeling of intense fatigue is also particularly common among the elderlies, who might be discouraged to persist on the physical trainings. Post exercise fatigue is in fact manifestations of "Oxidative Stress" resulting from the accumulation of metabolites produced during active muscular activities and micro damages. If the production of Reactive Oxygen Species (ROS) found in the skeletal muscles, blood and lipids, enhanced by the presence of free radicals during heavy exercises, can be reduced, "Oxidative Stress" could be alleviated. We intend to produce an "energizer", that nor only help to decrease "Oxidative Stress", but also provide essential nutritional support to the muscles as well as longer term joint space integrities. Verbascoside containing herb was chosen to be the antioxidant to release "Oxidative Stress". Creatine was the nutritive agent for muscle repair and collagen (related to Glucosamine) was added to provide cartilage support. The "energizer" was put to a properly planned clinical trial involving healthy young men. The study was designed as a randomized, double blinded cross-over study. All participants randomly consumed the energizer, collagen powder or creatine monohydrate for 14 days with a wash-out period interval for at least on month. On day 15 the participants completed one bout of well calibrated motor-driven treadmill exercise. Monitoring consisted of heart rate, and blood samples for the measurements of oxidative stress biomarkers, and muscle soreness. Creatine Kinase was measured in the subsequent recovery days. Results showed that the "energizer" produced a decreasing trend in the metabolites of proteins, DNA and lipids, and muscle soreness significantly decreased and during the three recovery days, Creatine Kinase activity sharply decreased, compared with the taking of collagen or creatine alone. In conclusion, the positive result supports the expectation that the "energiser" could be safely used as an exercise supplement for the prevention of after exercise fatigue.
\end{abstract}

Keywords: Energiser, Verbascoside, Musculo-Skeletal Supplement

\section{Introduction}

In the modern communities, the practice of bodily exercises as means to maintain physical strength and general health state has become important components of peoples' day to day livelihood. At the same time, increasing human longevity is complicated by aging related physiological deteriorations $[1,2]$. People approaching senior ages are more concerned with means to maintain health and longevity. Practice of physical exercises hence has become more important for the elderlies $[3,4]$.
Among the city-dwellers, most people making serious efforts to maintain a regular practice of exercises are sedentary workers. One common complaint among those who return from strenuous exercises is the feeling of intense fatigue. The elderlies are even more severely affected. We understand today that the so called exercise fatigue is in fact manifestations of "oxidative stress" which takes time to recover $[5,6]$. Aging is known to alter the neurohormonal mechanisms that control the cardiovascular system, manifested as a decline in sinoatrial node parasympathetic 
activity and an increase in sympathetic activity in the heart and vascular system [7].

The post exercise fatigue often discourages the individual, particularly the elderlies, from carrying on regular physical training. The practical way to overcome this difficulty is to formulate a gradually intensifying program so that over-training could be avoided. At the same time, the nutritional aspects should also be carefully looked into: Is the fatigue related to nutritional deficiencies? Is there nutritional supplements that could help with the maintenance of muscle strength? Is there nutritional supplement that would quickly remove the oxidative stress? Is there a need to provide longer term musculo-skeletal integrity?

\section{Creating an Energiser}

The aim of producing a supplement to specifically prevent or overcome oxidative stress should not be orientated towards the boostering up of physical performance, (which is important only for the professional athletes), but rather to bring about a smooth recovery after strenuous heavy exercises. Ideally, the Energiser is composed of three types of supplement, viz., a muscle nutrient, an antioxidant and a provider for long-term cartilage health.

\subsection{Nutritional Component}

During muscular exercises, the acute demand for energy requires adequate oxygen consumption which could not be maintained in a perfect balance, resulting in the accumulation of free radicals. The imbalance between the free radicals which are hurting the anti-oxidant defenses and muscle tissues results in the oxidative stress [8]. Muscles tissues should be kept in a perfect nutritional state in order to be able to resist the challenge.

Nutritional components related to muscle tissues are plentiful. Carbohydrate related substances tend to provide short term support to muscle strength $[9,10]$. On a long term need for muscular support, nutritional items related to the muscle substance itself could be a good choice. Creatine is an essential amino acid heavily contained in muscles. Taking creatine as a food supplement has been practiced among those patients recovering from chronic illnesses. Patients put on long term casting because of long bone fractures when muscle training becomes impossible, are put on creatine so that after removal of the rigid cast, muscle strength recovery can be hastened $[11,12]$.

In our creation of the Energiser, therefore, Creatine is chosen as a component.

\subsection{Anti-oxidant Component}

While physical fatigue could be related to an accumulation of free radicals resulting from a deficient anti-oxidant system, it has also been found that at the same time, there could be a suppression of the immune system, such as reduced proliferative activity of splenocytes and high $\mathrm{Th} 1 / \mathrm{Th} 2$ ratio which lead to the secretion of specific subsets of cytokines unfavourable for physiological balance and disease prevention
$[13,14]$.

Phytoestrogenes have been studied and found to have enhancing effects on antioxidant enzymes and modulate sex hormone levels among the females $[15,16]$. Dietary supplementation with phytoestrogens has been found to enhance the glutathione-dependent enzyme activities in erythrocytes in response to short intense exercise $[16,17]$. Among the plant derived chemical compounds, Verbascoside has been widely reported to be a potent anti-oxidant. Verbascoside has been found in a number of plants, viz., Lippa citriodora [16], Lemon Verbena and Buddleja officialis [19]. The latter is found in the wilder land of South-West China and carries a folk name of "Champion Horse Grass" implying that the horse runs faster after eating the grass.

Verbascoside has been found to improve the anti-oxidant capacity and prevent exercise-induced muscle fatigue and micro-injury in animal models [20, 21]. Female swimmers in their swimming exercises have experienced less muscle fatigue, quicker recovery and reduced levels of oxidation markers [16].

In our attempt to create an Energiser, we have chosen a common medicinal plant Buddaleja officialis, as the source of Verbascoside, the anti-oxidant.

\subsection{Protection of Musculo-Skeletal Integrity}

It is already a matter of beyond doubt that physical training protects health and is particularly relevant to the cardiovascular system [22]. For the seniors, exercise training for better cardiovascular health is even more important [23, 24]. However, senior people are often troubled by degenerative diseases of the joints and their excessive use in physical training might lead to progressive deteriorations. Indeed this dilemma of over-use of joints often prevents the persistence of exercises.

As the energiser is being planned, we are aware of the dilemma which prompts us to consider adding a component that may possibly protect the continuous deterioration of the cartilaginous joint surfaces.

Glucosamine is a structural component of cartilage, the tissue that forms the contact surfaces of a synovial joint. Glucosamine is produced naturally in the body. A yet unproven belief is that when glucosamine is orally taken, it helps to maintain the integrity of the cartilage surfaces via supportive regenerative processes. Glucosamine has been popular among the aging population who suffers from osteoarthritis of the weight bearing joints, particularly the knees, and the elderlies expect to enjoy a control of pain when walking. Many proper clinical trials on the efficacy of glucosamine in the control of osteoarthritic knee pain had been done [25] but the most extensive one is the Glucosamine/Choidroitin Arthritis Intervention Trial (GAIT). The GAIT study covered over 1500 clients over the age of 40 diagnosed as osteoarthritis for a period of 24 weeks, extending to 24 months. The results showed that all clients who suffered from mild knee pain did not improve better than placebo, either on 24 weeks short term or 24 months long term follow-up [26]. One has to realize that osteoarthritis of the 
knees involves non-inflammatory degenerations resulting from repetitive uses, minor injuries and deformities and the pain is felt mostly on mobilization. It is therefore logical to find that glucosamine did not compare well with any pain-relieving agent. Any demonstration of improvement unrelated to mobilization (i.e. walking) with structural evidences might be a better way to assess the efficacy of glucosamines. It is interesting to note that Chondroitin used for six months was found to improve pain and function of osteoarthritic hands [26]. Some European studies also found that glucosamine improved knee pain and function while at the same time slowed down the progression of arthritis [27]. Both studies looked at the progressive narrowing of the joint spaces under treatment for three years and found that the deterioration was milder among the treatment group.

With such impressive observations, one may confidently take glucosamine as an agent of preventive value rather than for treatment and include it as a component of the Energiser.

\section{Clinical Study to "Validate" the Relieving Effects of the Energiser on Oxidative Stress}

The Energiser was composed of the nutritional part, creatine, the herbal anti-oxidant verbascoside contained in the standard extract of Buddleja officinalis, and the cartilage supporting glucosamine contained in the less sophisticated product of collagen. The study involved eight healthy young men who took 14 day's supplements, then participated in a well-organized eccentric exercise program. The age, height, weight, body mass index and body fat percentage of participant were $20.9 \pm 1.0$ year, $174.0 \pm 4.0 \mathrm{~cm}, 65.1 \pm 8.8 \mathrm{~kg}$, $21.6 \pm 2.4 \mathrm{~kg} / \mathrm{m}^{2}$ and $17.3 \pm 3.7 \%$.The oxidative stress and micro-damages of the muscle were studied.

The study was done at the Department of Sports Science and Physical Education at the Chinese University Of Hong Kong during Jun 2012 to Mar 2013, ethical approval was obtained prior to the trial commencement from the Clinical Research Ethics Committee of the Chinese University of Hong Kong.

During the experimental period, participants were instructed to follow their routine living schedule and dietary habits. All participants were required to refrain from alcohol consumption and vigorous physical activities 24 hours before the test. A two-day weighed food record diary was obtained from each participant. Participants were requested to repeat the same diet before the next trials. During the trial, participants were asked to avoid anti-inflammatory drugs, pain-relieving ointments or creams, exercises, stretchings or massage.

A calibrated motor-driven treadmill was used. All participants were required to complete one preliminary test to determine the relationship between downhill running speed and heart rate (HR). The speed was chosen with reference to each participant's training status and was set between $60 \%$ and $90 \%$ of the individual age predicted maximal HR
(HRmax $=220$-age). The downhill running speed which was equivalent to around $75 \%$ of individual HRmax was determined. All participants then undertook $30 \mathrm{~min}$ of downhill running at the predicted speed to confirm, and if necessary, to adjust the running speed for the main trials.

The study was designed as a randomized, double-blinded cross-over study. All participants randomly consumed herbal Engergizer (HE), collagen powder (CP), or creatine monohydrate (HM) sachet daily in the morning $(11.5 \mathrm{~g}$ per sachet) for 14 days with a wash-out period interval for at least one month. On day 15, the participants completed one bout of exercise and followed by three recovery days. The packing and weight of the sachet is identical, both the participants and investigators were blinded to the contents of the sachet given to each participant.

On Day 1 morning, the baseline data, i.e., body weight, body height, muscle soreness score, venous and capillary blood samples, were collected. Then the participants were given one of three supplements. On Day 15 morning, all participants reported to the lab and ingested around $500 \mathrm{ml}$ of distilled water to ensure the hydration status. On arrival, subjects rested for $20 \mathrm{~min}$ before the insertion of the cannula into the antecubital vein in the forearm. A standardized $5 \mathrm{~min}$ warm-up at $50 \%$ of individual HRmax was performed after the collection of the blood samples. The speed of treadmill was then increased to intensity of $70 \%$ of individual HRmax immediately after warm-up session and subjects completed down-hill running $(-16.5 \%)$ for $45 \mathrm{~min}$ at such exercise intensity [28]. Heart rate was monitored and recorded throughout the exercise. Further capillary samples were taken during each exercise bout at regular time points, whereas venous and capillary samples were obtained immediately after the down-hill running. On the morning of three recovery days, similar data were collected. Three-way mixed, repeated measures ANOVA, ANCOVA and MANOVA (supplements $\mathrm{x}$ trial sequence $\mathrm{x}$ time) were used to do data analysis.

\subsection{Measurement}

Venous blood samples were used to assess oxidative stress biomarkers, i.e., 8-isoprostane, protein carbonyls, and 8-hydroxy-2'-deoxyguanosine (8-OHdG), using commercial EIA kits or Colorimetric Assay kit. Capillary blood samples were used to test Creatine Kinase (CK) level (Reflontron ${ }^{\circledR}$, Sweden). Likert scale was used to assess muscle soreness [29]. Rating of abdominal discomfort (RAD) was assessed on a 10-point scale, where 1 was "completely comfortable" and 10 was "very uncomfortable" [30]. Rating of perceived exertion (RPE) was estimated using the 6- to 20-point Borg scale [31]. Proper statistical analysis was completed.

\subsection{Result}

Immediately after exercise, the $8-\mathrm{OHdG}$ concentration increased in CP trial, but not $\mathrm{HE}$ and $\mathrm{CM}$ trials. There was a trend of lower 8-OHdG concentration in the HE trial than that in the $\mathrm{CP}$ trial $(\mathrm{P}=0.062)$. Comparing with the $8-\mathrm{OHdG}$ 
concentration $2 \mathrm{hr}$ after exercise, a continual decrease of 8 -OHdG $(1.9 \%-2.3 \%)$ was observed in the HE trial during the following two recovery days. However, increases of 8-OHdG were observed in the $\mathrm{CP}$ and $\mathrm{CM}$ trials, with only an exception of a decrease on day 17 (D17) in the CP trial. The increase of 8 -OHdG in the CM trial, attained statistical significance $(\mathrm{p}=0.049)$. Although other changes did not attain statistical significance, the trend of the HE producing more on $8-\mathrm{OHdG}$ decline was obvious.

\subsubsection{Protein Carbonyls (PC)}

There was a marked trend of decrease in Protein carbonyls (PC) during recovery in the HE trial although it did not reach statistical significance $(\mathrm{p}=0.203)$. However, no such trends were noticed in $\mathrm{CP}$ and $\mathrm{CM}$ trials.

\subsubsection{Creatine Kinase (CK)}

Creatine kinase activity peaked at REC-1d and decreased in all trials.

\subsubsection{Muscle soreness scores}

There was a significant decrease $(56.5 \%)$ in muscle soreness scores in the HE trial $(\mathrm{p}=0.001)$, while no such significant changes were noticed in the $\mathrm{CP}$ and $\mathrm{CM}$ trials $(\mathrm{p}=0.488$ and $\mathrm{p}=0.153$, respectively).

\section{Discussion}

Strenuous physical exercise can stimulate the production of reactive oxygen species, which elevate markers of oxidative stress in the skeletal muscle, blood, and lipids of athletes [32]. Free radicals could increase the concentrations of the metabolites of these bio-molecules in the blood, including 8-OHdG, 8-isoprostane, and Protein Carbonyls. Elevation of 8-isoprostane content indicates an increase in the rate of whole body lipid peroxidation [33]. A decrease of 8-OHdG indicates a reduction of oxidative damage to DNA [34]. The energiser study demonstrated a remarkable decreasing trend in the metabolites of proteins, DNA and lipids after the oral administration of the Buddleja officinalis containing formula.

The CK is an enzyme located mainly in skeletal muscles. Its activity in plasma is the index of muscle fibers injuries [35]. The CK concentrations peaked $24 \mathrm{hrs}$ after exercise in all three trials, which indicated that significant muscle micro-damage were observed in the study. The formula containing Buddleja officinalis sharply decreased CK activity during three recovery days when compared with other formulae. Therefore, it is obvious that the Energizer is more effective in decreasing $\mathrm{CK}$ concentration when compared with collagen or creatine.

The results of the present study showed that the formulation containing Buddleja officinalis significantly reduced muscle soreness after the exercise, while no significant effects were observed in the non-Buddleja containing formulations.

It should be noted that the creatine content in the Energiser formula may also contribute to the protective effects. One recent study indicated that creatine supplementation could also reduce oxidative DNA damage and lipid peroxidation induced by a single bout of resistant exercise [36].

In conclusion the major finding of the Energiser trial showed that the Energiser has potential effects on improving the recovery of oxidative damages and muscle microdamages caused by strenuous eccentric exercises in healthy young adults. The positive results well supports the expectation that this exercise supplement could be useful in the prevention of after exercise fatigue. For the elderlies who are frequently bothered by fatigue even after normal activity exercises, the energiser could be a genuine guarantee to maintain their resilience on keeping an active life.

\section{Acknowledgements}

Authors are indebted to the Ming Lai Foundation support given to the team responsible for the study.

\section{References}

[1] W. B. Cannon, The wisdom of the body, 1st ed. New York: W.W. Norton \& Company, 1932.

[2] M. Esler, J. Hastings, G. Lambert, D. Kaye, G. Jennings, D. R. Seals. The influence of aging on the human sympathetic nervous system and brain norepinephrine turnover. Am J Physiol Regul Integr Comp Physiol. 2002, 282(3): R909-916.

[3] J. R. Stratton, W. C. Levy, J. H. Caldwell, A. Jacobson, J. May, D. Matsuoka, et al. Effects of aging on cardiovascular responses to parasympathetic withdrawal. J Am Coll Cardiol. 2003, 41(11): 2077-2083.

[4] E. F. Binder, S. J. Birge, R. Spina, A. A. Ehsani, M. Brown, D. R. Sinacore, et al. Peak aerobic power is an important component of physical performance in older women. J Gerontol A Biol Sci Med Sci. 1999, 54(7): M353-356.

[5] J. Finaud, G. Lac, E. Filaire E. Oxidative stress: relationship with exercise and training. Sports Med. 2006, 36(4): 327-58.

[6] K. Fisher-Wellman, R. J. Bloomer. Acute exercise and oxidative stress: a 30 year history. Dyn Med. 2009, 8:1.

[7] A. L. Mark. Sympathetic dysregulation in heart failure: mechanisms and therapy. Clin Cardiol. 1995, 18(3 Suppl I): I3-8.

[8] P. Montuschi, P. J. Barnes, L. J. Roberts. Isoprostanes: markers and mediators of oxidative stress. FASEB J. 2004, 18(15): $1791-1800$.

[9] Y. J. Chen, S. H. Wong, C. K. Wong, C. W. Lam, Y. J. Huang, P M. Siu. The effect of a pre-exercise carbohydrate meal on immune responses to an endurance performance run. Br J Nutr. 2008, 100(6): 1260-1268.

[10] Y. J. Chen, S. H. Wong, C. O. Chan, C. K. Wong, C. W. Lam, P. M. Siu. Effects of glycemic index meal and CHO-electrolyte drink on cytokine response and run performance in endurance athletes. J Sci Med Sport. 2009, 12(6): 697-703.

[11] R. Jäger, M. Purpura, A. Shao, T. Inoue, R. B. Kreider. Analysis of the efficacy, safety, and regulatory status of novel forms of creatine. Amino Acids. 2011, 40(5): 1369-1383. 
[12] M. A. Tarnopolsky. Caffeine and creatine use in sport. Ann Nutr Metab. 2010, 57 (Suppl 2): 1-8.

[13] D. R. Lucey, M. Clerici, G. M. Shearer. Type 1 and type 2 cytokine dysregulation in human infectious, neoplastic, and inflammatory diseases. Clin Microbiol Rev. 1996, 9(4): $532-562$.

[14] M. Lyall, M. Peakman, S. Wessely. A systematic review and critical evaluation of the immunology of chronic fatigue syndrome. J Psychosom Res. 2003, 55(2): 79-90.

[15] G. G. Kuiper, J. G. Lemmen, B. Carlsson, J. C. Corton, S. H.Safe, P. T. van der Saag, et al. Interaction of estrogenic chemicals and phytoestrogens with estrogen receptor beta. Endocrinology. 1998, 139(10): 4252-4263.

[16] A. Mestre-Alfaro, M. D. Ferrer, A. Sureda, P. Tauler, E. Martínez, M. M. Bibiloni, et al. Phytoestrogens enhance antioxidant enzymes after swimming exercise and modulate sex hormone plasma levels in female swimmers. Eur J Appl Physiol. 2011, 111(9): 2281-2294.

[17] H. Adlercreutz, C. Bannwart, K. Wähälä, T. Mäkelä, G Brunow, T. Hase, et al. Inhibition of human aromatase by mammalian lignans and isoflavonoid phytoestrogens. J Steroid Biochem Mol Biol. 1993, 44(2): 147-153.

[18] L. Funesa, S. Fernández-Arroyoa, O. Laportaa, A. Ponsb, E. Rochec, A. Segura-Carreterod, et al. Correlation between plasma antioxidant capacity and verbascoside levels in rats after oral administration of lemon verbena extract. Food Chemistry. 2009, 117(4); 589-598.

[19] H. S. Wong, F. H. Sun, Y. J Huang, S. Y. Pang, K. F. Cheng, P. C. Leung, et al. Effects of a herbal supplement to protect healthy males from oxidative stress and muscle micro-damage during eccentric exercise: A pilot study. International Journal of Food and Nutrition. (in press).

[20] M. J. Liu, J. X. Li, H. Z. Guo, K. M. Lee, L. Qin, K. M. Chan. The effects of verbascoside on plasma lipid peroxidation level and erythrocyte membrane fluidity during immobilization in rabbits: a time course study. Life Sci. 2003, 73(7): 883-892.

[21] F. Liao, R. L. Zheng, J. J. Gao, Z. J. Jia. Retardation of skeletal muscle fatigue by the two phenylpropanoid glycosides: verbascoside and martynoside from Pedicularis plicata maxim. Phytother Res. 1999, 13(7): 621-623.

[22] K. D. Monahan, F. A. Dinenno, H. Tanaka, C. M. Clevenger, C A. DeSouza, D. R. Seals. Regular aerobic exercise modulates age-associated declines in cardiovagal baroreflex sensitivity in healthy men. J Physiol. 2000, 529(Pt 1): 263-271.

[23] J. N. Morris, A. E. Hardman. Walking to health. Sports Med. 1997, 23(5): 306-332.
[24] P. K. Stein, A. A. Ehsani, P. P. Domitrovich, R. E. Kleiger, J. N. Rottman. Effect of exercise training on heart rate variability in healthy older adults. Am Heart J. 1999, 138(3 Pt 1): 567-576.

[25] N. Poolsup, C. Suthisisang, P. Channark, W. Kittikulsuth. Glucosamine long-term treatment and the progression of knee osteoarthritis: systematic review of randomized controlled trials. Ann Pharmacother. 2005, 39(6): 1080-1087.

[26] A. D. Sawitzke, H. Shi, M. F. Finco, D. D. Dunlop, C. L. Harris, N. G. Singer NG, et al. Clinical efficacy and safety of glucosamine, chondroitin sulphate, their combination, celecoxib or placebo taken to treat osteoarthritis of the knee: 2-year results from GAIT. Ann Rheum Dis. 2010, 69(8): 1459-1464.

[27] K. Pavelká, J. Gatterová, M. Olejarová, S. Machacek, G. Giacovelli, L. C. Rovati. Glucosamine sulfate use and delay of progression of knee osteoarthritis: a 3-year, randomized, placebo-controlled, double-blind study. Arch Intern Med. 2002, 162(18): 2113-2123.

[28] M. I. Kingsley, L. P. Kilduff, J. McEneny, R. E. Dietzig, D. Benton. Phosphatidylserine supplementation and recovery following downhill running. Med Sci Sports Exerc. 2006, 38(9): 1617-1625.

[29] D. M. High, E. T. Howley, B. D. Franks. The effects of static stretching and warm-up on prevention of delayed-onset muscle soreness. Res Q Exerc and Sport. 1989, 60(4): 357-361.

[30] T. D. Noakes. Fluid replacement during exercise. Exerc Sport Sci Rev. 1993, 21: 297-330.

[31] G. A. Borg. Perceived exertion: a note on "history" and methods. Med Sci Sports. 1973, 5(2): 90-93.

[32] P. M. Clarkson. Antioxidants and physical performance. Crit Rev Food Sci Nutr. 1995, 35(1-2): 131-141.

[33] H. J. Kim, C. Jamart, L. Deldicque, G. L. An, Y. H. Lee, C. K. $\mathrm{Kim}$, et al. Endoplasmic reticulum stress markers and ubiquitin-proteasome pathway activity in response to $200-\mathrm{km}$ run. Med Sci Sports Exerc. 2011, 43(1): 18-25.

[34] A. Valavanidis, T. Vlachogianni, C. Fiotakis. 8 - hydroxyl - 2' - deoxyguanosine (8-OHdG): a critical biomarker of oxidative stress and carcinogenesis. J Environ Sci Health C Environ Carcinog Ecotoxicol Rev. 2009, 27(2): 120-139.

[35] H. Kuipers. Exercise-induced muscle damage. Int J Sports Med. 1994, 15(3): 132-135.

[36] R. Rahimi. Creatine supplementation decreases oxidative DNA damage and lipid peroxidation induced by a single bout of resistance exercise. J Strength Cond Res. 2011, 25(12): 3488-3455. 